\title{
An integrated assessment of particulate respirators used as personal protection from ambient air pollution in Bangkok, Thailand
}

\author{
Kennedy L McGuinness', Isabella R Hinks ${ }^{1}$, Katherine E Westcott', Shabbir H Gheewala ${ }^{2}$ (1) \\ 1 Institute for the Environment, University of North Carolina at Chapel Hill (UNC-CH), Chapel Hill, North Carolina, USA; The Joint Graduate School of \\ Energy and Environment (JGSEE), King Mongkut's University of Technology Thonburi, Bangkok, Thailand, ${ }^{2}$ The Joint Graduate School of Energy and \\ Environment (JGSEE), King Mongkut's University of Technology Thonburi, Bangkok, Thailand; Center of Excellence on Energy Technology and \\ Environment, PERDO, Ministry of Higher Education, Science, Research and Innovation, Bangkok, Thailand; Department of Environmental Sciences and \\ Engineering, Gillings School of Public Health, University of North Carolina, Chapel Hill, North Carolina, USA \\ Keywords: thailand, respirator, ambient air pollution, particulate matter
}

https://doi.org/10.29392/001c.14598

\section{Journal of Global Health Reports}

Vol. 4, 2020

\begin{abstract}
The recent increase of fine particulate matter in Bangkok, Thailand has become a widespread public health concern. Wearing a particulate respirator is one method to reduce particulate inhalation and therefore mitigate the adverse health effects of ambient air pollution. This study provides an integrated assessment of seven particulate respirator models based on three criteria: effectivity, cost, and environmental impact. The overall effectivity of each model is evaluated by assessing the product's features associated with facial fit. The cost criterion reflects current market prices for bulk and individual orders. Thirdly, an environmental impact score is determined for the product life cycle of each respirator using life cycle assessment. The study assesses each respirator as it would be manufactured, distributed, used, and disposed of in Bangkok, Thailand. The integrated assessment results in twelve distinct consumer frameworks, reliant on variations of the three criteria, to provide guidance for policy makers and independent consumers in the selection of particulate respirators to optimally suit their needs. Ultimately, the data suggest that a buyer's ideal respirator choice for short-term use is a disposable particulate respirator with a head strap. For long-term use, the study recommends a low-cost reusable respirator with an exhalation valve and replaceable filters. The average effectivity of reusable respirators is found to be greater than that of disposable respirators, due to their higher average number of available sizes. Reusable respirators are associated with consistently lower environmental impacts. The short-term cost of disposable respirators is much less than those of reusable models, however prices do converge over time.
\end{abstract}

The annually-increasing presence of microscopic airborne particulates with a diameter equal to or greater than 2.5 microns (PM2.5) in Bangkok, Thailand has led to a rising concern for public health. The World Health Organization (WHO) states that the maximum safe level of annual average PM2.5 concentration is $10 \mu \mathrm{g} / \mathrm{m}^{3}$ or less. ${ }^{1}$ However, in 2018, the annual average concentration of PM2.5 in Bangkok and the surrounding vicinity was approximately 30 $\mu \mathrm{g} / \mathrm{m}^{3}$-well over the maximum safe concentrations as stated by the WHO. ${ }^{2}$ Concentrations continue to rise; the 2018 measurements were $20 \%$ higher than those recorded in 2017.3,4 The most prominent sources of PM2.5 that have led to this issue are vehicular emissions, industrial discharge in concentrated industrial zones, biomass burning and transboundary haze. ${ }^{5,6}$

Although the severity of chronic PM 2.5 exposure is still being investigated, several recent studies have begun to establish positive correlations between high levels of particulate air pollution and respiratory illness, cardiovascular disease, neuropsychological symptoms, overall hospitalisations, and increases in premature mortality. ${ }^{2,5,7,8}$ The
World Health Organization estimates that one third of stroke-, lung cancer-, and disease-related deaths in urban environments such as Bangkok are due to air pollution. ${ }^{9}$

While chronic exposure appears to affect all exposed members of the population, acute exposure to fine particulates has been found to disproportionately affect certain groups, including the elderly, young children, and persons with chronic cardiopulmonary disease, influenza, or asthma. ${ }^{8,10}$ For both children and asthmatics, fine particulate exposure leads to increased hospitalisations and respiratory symptoms. Although this exposure type may have minimum overall health consequences, it affects the day-to-day lives of residents, often causing short-term absence from work or school. ${ }^{11}$

One method of preventing the adverse effects of air pollution is to wear a particulate respirator ${ }^{12}$; respirators function as breathable barriers between the ambient air and a user's airways. Worn over the mouth and nose, they are airtight half-masks that block particulates and other harmful pollutants from entering the body. ${ }^{13,14}$ Different respirators have varying levels of overall efficiency, depending on their fit and filter media efficiency. ${ }^{5,15-17}$ 


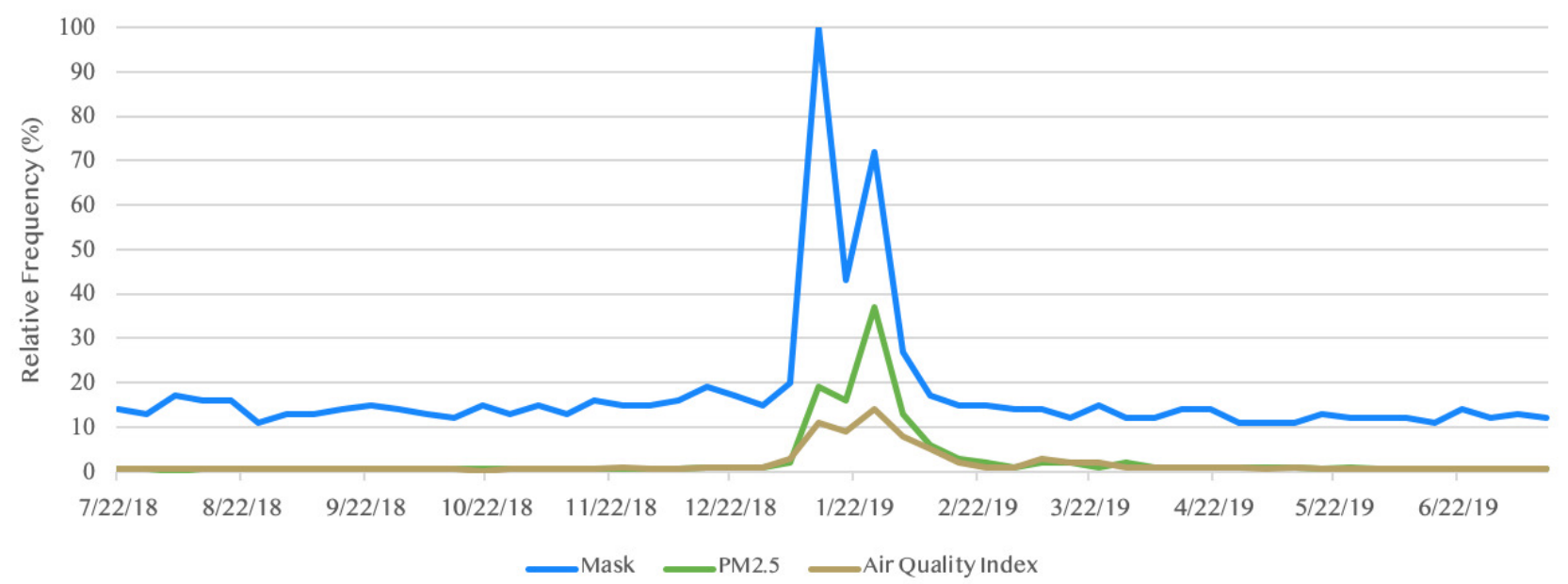

Figure 1. Relative frequency of google searches for topics "air quality index", "PM2.5" and "mask" in Bangkok.

The figure shows a prominent spike in searches for all of these keywords at the end of January 2019; this corresponds with one of the worst periods of ambient air pollution that Bangkok has seen to date.

An episode of extreme air pollution in January 2019 sparked a surge in Google searches relating to Bangkok's air quality indices and PM2.5 levels, visualized in Figure 1. ${ }^{18}$ The event inspired many residents of Bangkok to begin wearing respirators. However, many of the respirators worn by residents were insufficient at protecting them from PM2.5 and other air pollutants; this is largely due to a lack of awareness regarding appropriate respirator types and fit. A 2017 cross-sectional field study by Rutgers University found that $53 \%$ of young adult street vendors in Bangkok never wore respirators. ${ }^{19}$ None of those individuals using respirators were using breathing protection that was specifically designed for protection against PM10 or PM2.5; $71 \%$ of the respirator wearers used hygiene or surgical masks. ${ }^{19}$

Surgical masks fail to protect users at the same level as respirators that are specifically designed to trap and protect against fine particulate matter. Studies show that respirators that are officially certified to filter at least $95 \%$ of PM2.5 are about 8-15 times more efficient than surgical masks, in terms of penetration and fit testing. ${ }^{19-22}$ Additionally, surgical masks allow an average of $20 \%$ penetrance of ambient particles, compared to the 0.1-1.4\% penetrance allowed by masks manufactured specifically for protection against small particles. ${ }^{23}$ In the surge of residents who are beginning to take necessary steps to protect themselves from ambient air pollution, inefficient masks are providing a false sense of security.

If the street vendors surveyed in the Rutgers study are representative of the rest of the residents in Bangkok, it can be assumed that the majority of Bangkok's residents are not properly protected against PM2.5. In this influential time of residents' piqued interest and a lack of respirator education, the spread of information regarding effective particulate respirators has the potential to educate Bangkok policy makers and independent consumers about the importance of protecting oneself from ambient air pollution, and the vital steps one must take to do so. Hence, this study aims to design a framework to assess the performance of respirators for protection against ambient air pollution in
Bangkok, Thailand, and apply it to rate respirators on their performance in fit, cost and environmental impact.

\section{METHODS}

The particulate respirators selected for this study were scored using a two-step process, ultimately providing integrated scores for 12 separate consumer frameworks. Each framework was defined by a distinct set of consumer demands. The purpose of the exploratory assessment was to suggest optimal respirators to individual and bulk consumers with a range of needs and preferences. The overall methodology of this process is visualized in Figure 2.

Figure 3 displays the twelve frameworks and a breakdown of their design. In Step 1 of the scoring process, each respirator was scored according to three criteria: overall effectiveness, cost, and environmental impact. The separate criterion scores each fell on a five-point scale (0-5), with the highest scores given to respirators with high effectivity, low cost, and low environmental impact, respectively. The criterion scores were dependent on four distinct consumer scenarios, A-D, each characterized by two factors: order size, and duration of use. The study considered scenarios in which consumers wear respirators all year long, or only during episodes of extreme air pollution (referred to in this study as "haze periods"). The scenarios are: (A) individual order for a haze period, (B) individual order for a yearlong period, (C) bulk order for a haze period, and (D) bulk order for a year-long period. Each scenario therefore had three independent criterion scores based on these factors.

The second step of the scoring process was the aggregation of criteria scores within scenarios A-D, which were weighted with three consumer purchasing models in mind: low-cost purchasing, in which the cost criterion was weighted most significantly, sustainable purchasing, wherein the environmental impact criterion was weighted most significantly, and controlled purchasing, in which all criteria were weighted equally (Figure 4). 


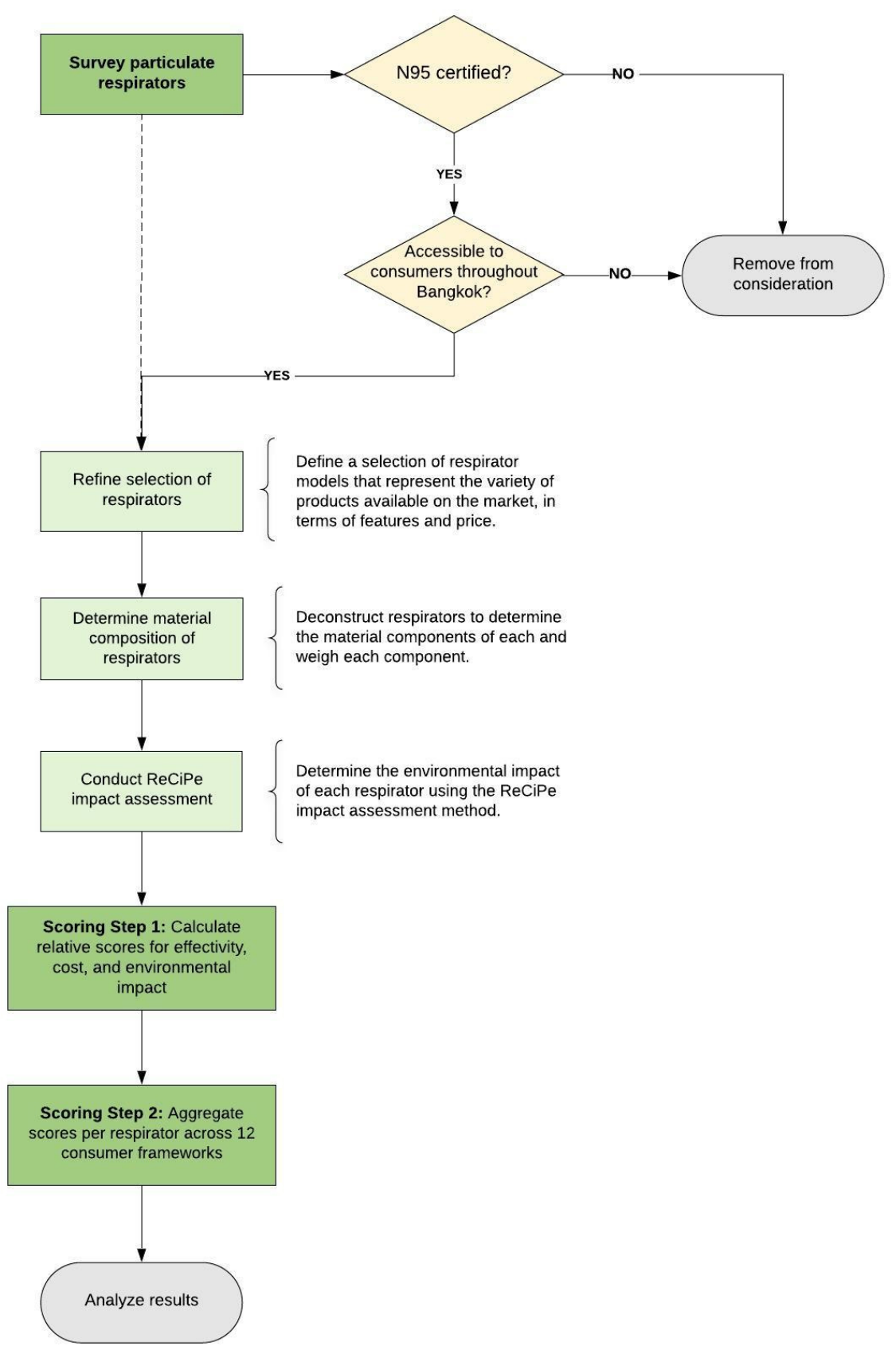

Figure 2. Flowchart of methodological approach.

The figure offers a visualization of the steps taken to select particulate respirators that reflect the spectrum of particulate respirators available to consumers in Bangkok, Thailand, and assess their relative scores across 12 consumer frameworks.

\section{RESPIRATOR SELECTION}

The seven particulate respirator models reported in this study were chosen based on their filtration effectiveness, accessibility to the average consumer in Bangkok, Thailand, and representation of the total selection of particulate respirators that are available in the Bangkok metropolitan area. Each respirator has been lab certified to filter at least $95 \%$ of particles greater than 0.3 microns by a standardized particle filtration effectiveness test, and can be readily found in local pharmacies, health stores, convenience stores, or shipped to any address in the Bangkok metropolitan area. The respirators included in the study span the spectrum of common respirator prices in Bangkok, and include a variety of common positioning properties, to reflect the range of respirators available on the market. As it is not the purpose of this study to promote any specific respirator manufacturer, nor is this study affiliated with any respirator manufacturer, the manufacturer names of each respirator model considered in this study are not disclosed. Rather, it is the focus of this study to analyze each respirator model based on its features and material components.

There are two respirator classifications: disposable and reusable. While disposable respirators are made for shortterm particulate filtration, reusable respirators are capable 


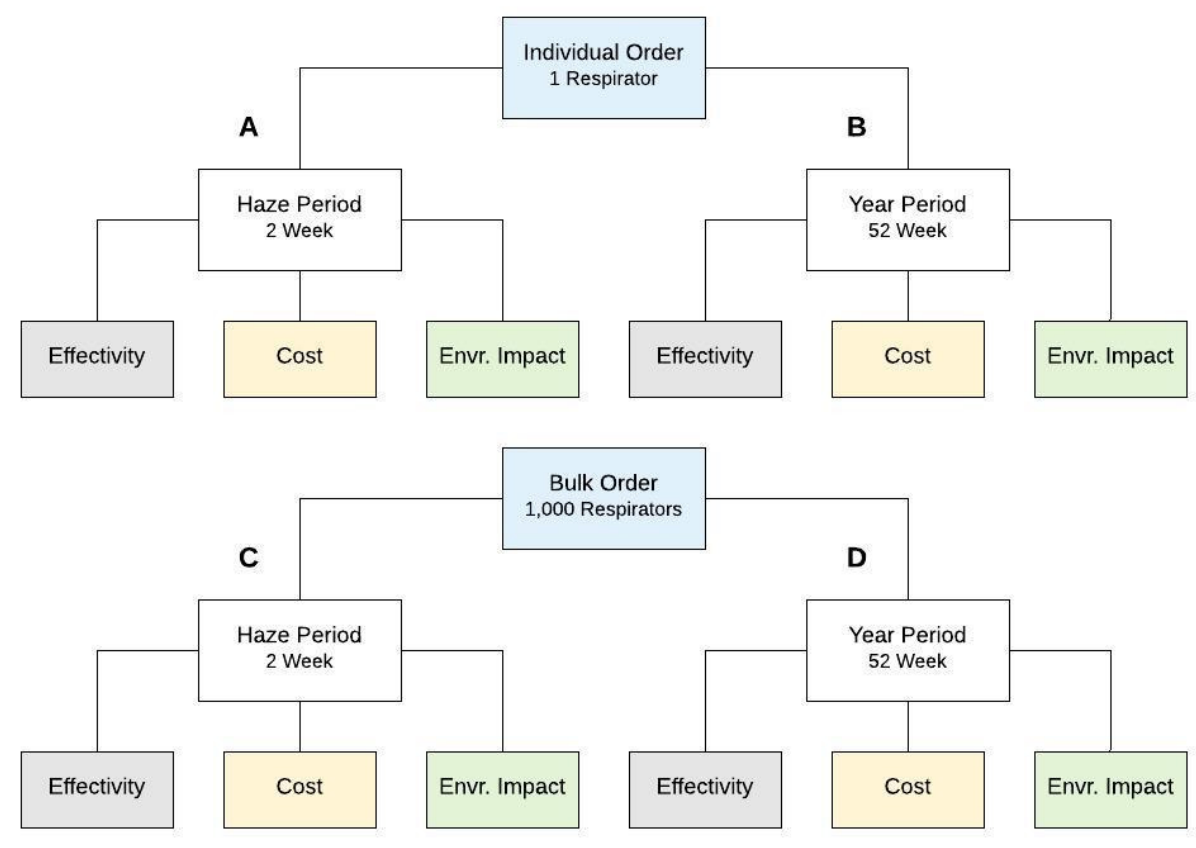

Figure 3. Twelve consumer frameworks.

Dependence of the three criteria scores (effectivity, cost, and environmental impact) on a specific consumer scenario. The consumer scenarios (A-D) are characterized by one of two order sizes (individual or bulk) and one of two time periods (haze or year).

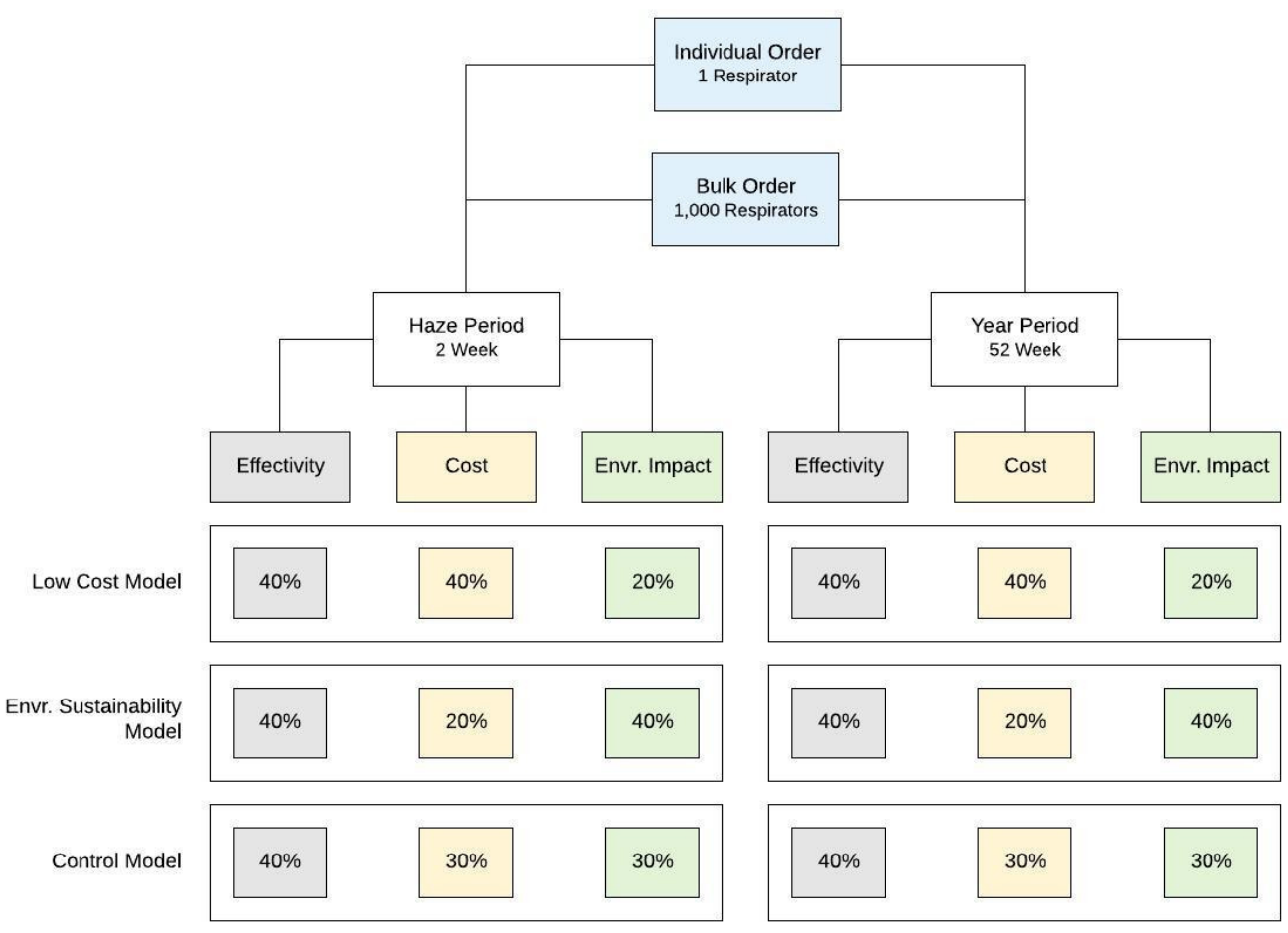

Figure 4. Consumer purchasing models in scenarios A-D.

This diagram illustrates the application of the three consumer purchasing models (control, low-cost, and sustainability) to the four consumer scenarios. This results in 12 distinct consumer frameworks.

of protecting the user for a longer term, if given proper care. tors from their disposable counterparts, and impact their Several structural features differentiate particulate respira- overall effectiveness; the inclusion or exclusion of these 
features was considered in the effectivity scoring criterion. Any respirator that has not specifically been developed to block the inhalation of particulate matter, such as a surgical mask, was not considered in this study, as the effectiveness of these masks tend to be extremely limited when they are used as respirators.

Three of the respirators considered in this study recommend the replacement of removable filters, which increase the longevity of the respirator shell while maintaining the efficiency of the respirator, itself. The filters were acknowledged in the data, contributing to both the cost and environmental impact criteria scores.

\section{LONGEVITY FACTOR}

The longevity factor is a functional unit that represents the amount of time that a given particulate respirator can provide protection before it must be replaced. The longevity of a given particulate respirator depends largely on the commute time, work type, and lifestyle of the individual who utilizes it, as well as the air quality of that individual's environment. Because of these per capita variations, respira- tor manufacturers are unable to suggest exact temporal use limitations; rather they provide use recommendations. The longevity factor of each respirator was estimated in units of weeks, based on these manufacturer recommendations. Longevity factors were used to determine the total number of respirators or filters that must be purchased within the specified time period.

As represented in Table 1, respirator models RR1-RR4 represent four separate reusable respirator types with varying features such as replaceable filters and exhalation valves. These models can be found online and in store. The disposable respirator models DR1-DR3 represent more general disposable respirator types sold in Bangkok pharmacies and convenience stores. DR1 is a hard-shell cup respirator without an exhalation valve, DR2 is a hard-shell cup respirator with an exhalation valve, and DR3 is a flat-fold respirator with an exhalation valve. To determine the scores of the DR1-DR3 models, variants of each model from leading manufacturers were assessed, and their respective scores were subsequently averaged. 
Table 1. Particulate respirator models and features

\begin{tabular}{|c|c|c|c|c|c|c|c|c|c|}
\hline $\begin{array}{l}\text { Respirator model } \\
\text { ID }\end{array}$ & Type & $\begin{array}{c}\text { Replaceable } \\
\text { filters }\end{array}$ & $\begin{array}{c}\text { Available } \\
\text { sizes }\end{array}$ & $\begin{array}{l}\text { Head } \\
\text { strap }\end{array}$ & $\begin{array}{c}\text { Exhalation } \\
\text { valve }\end{array}$ & $\begin{array}{c}\text { Longevity } \\
\text { factor }\end{array}$ & $\begin{array}{l}\text { Upfront cost } \\
\text { (THB) }\end{array}$ & $\begin{array}{l}\text { Upfront cost } \\
\text { (USD) }\end{array}$ & Style \\
\hline RR1 & Reusable & $\checkmark$ & 4 & $\sqrt{ }$ & $\checkmark$ & 104 & 2,000 & 64.81 & $\begin{array}{l}\text { Flat } \\
\text { fold }\end{array}$ \\
\hline RR2 & Reusable & $\sqrt{ }$ & 4 & $v$ & $v$ & 52 & 400 & 12.96 & $\begin{array}{l}\text { Flat } \\
\text { fold }\end{array}$ \\
\hline RR3 & Reusable & & 3 & & $\checkmark$ & 20 & 1,300 & 42.12 & $\begin{array}{l}\text { Flat } \\
\text { fold }\end{array}$ \\
\hline RR4 & Reusable & $\checkmark$ & 2 & v & & 104 & 1,700 & 55.09 & Cup \\
\hline DR1 & Disposable & & $1-2$ & v & & 1 & 30 & 0.97 & Cup \\
\hline DR2 & Disposable & & $1-2$ & 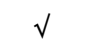 & v & 1 & 50 & 1.62 & Cup \\
\hline DR3 & Disposable & & 1 & $v$ & $\checkmark$ & 1 & 170 & 5.51 & $\begin{array}{l}\text { Flat } \\
\text { fold }\end{array}$ \\
\hline
\end{tabular}


Table 2. Consumer scenarios A-D.

\begin{tabular}{|l|l|l|}
\hline & 1 Respirator & 1,000 Respirators \\
\hline Haze period & $\begin{array}{l}\text { (A) Independent consumer to } \\
\text { order 1 particulate respirator for } \\
\text { personal use, with replacement as } \\
\text { needed, during a period of extreme } \\
\text { ambient air pollution. }\end{array}$ & $\begin{array}{l}\text { (C) Federal agency/organization } \\
\text { to bulk order particulate respirators } \\
\text { for large-scale distribution, with } \\
\text { replacement as needed, during a period } \\
\text { of extreme air pollution. }\end{array}$ \\
\hline Year-long period & $\begin{array}{l}\text { (B) Independent consumer to order } \\
\text { 1 particulate respirator for } \\
\text { personal use, with replacement as } \\
\text { needed, over the course of 1 year. }\end{array}$ & $\begin{array}{l}\text { (D) Federal agency/organization } \\
\text { to bulk order particulate respirators } \\
\text { for large-scale distribution, with } \\
\text { replacement as needed, over the } \\
\text { course of 1 year. }\end{array}$ \\
\hline
\end{tabular}

\section{CONSUMER SCENARIOS}

As Bangkok is an urban environment affected by moderate levels of PM2.5 year-round, ${ }^{23}$ some sensitive groups may seek year-round particulate protection. Other consumers may only seek particulate protection during short episodes of extreme air pollution. The study therefore devised four distinct consumer scenarios to represent the varied needs and interests of residents in Bangkok, Thailand.

No standards currently exist to define an episode of extreme air pollution, or "haze period;" this study defined a haze period as two consecutive weeks in which at least $1 /$ 3 of the total days have an air quality index (AQI) of 151 or greater, or a concentration of 55.5 micrograms per meter squared. The World Air Quality Index Project considers this index value to be an "unhealthy" level of air pollution for all individuals. ${ }^{24}$

Between the months of August 2018 and July 2019, Bangkok experienced three haze periods: the first period taking place in December 2018, and the second and third in January 2019.23 During this time, the government distributed masks and information about particulates to the public. ${ }^{25}$ Although mask distribution was limited to a small portion of Bangkok's population, it confirmed the Thai government's efforts and ability to combat the public health crisis. Additionally, the independent consumption of antipollution masks (particulate respirators) tends to increase significantly with AQI value increases, such as during haze periods. ${ }^{26}$ This integrated assessment operates under the assumption that consumer reactions will be similar in future AQI spikes in Thailand. The four consumer scenarios summarized in Table 2 were designed to distinctly consider "bulk" consumers, such as the Thai government buying respirators for population groups, and "individual" consumers, such as the average Thai citizen.

\section{STEP 1: CRITERIA SCORING}

After the establishment of the four consumer scenarios, criteria scoring was executed to assess the effectivity, cost, and environmental impact of each respirator model.

\section{EFFECTIVITY}

The effectivity of a particulate respirator on a given person is dependent on the filtration media used, as well as the fit. ${ }^{13,14}$ The filtration media used in all seven respirator models in this study are considered equally effective, as each medium filters fine particulates with at least $95 \%$ efficiency. Effectivity scores were thus scored by the material features of a respirator that are understood to offer the optimal fit.

The features of a respirator that optimize fit are size availability and the inclusion of a head strap or an exhalation valve. ${ }^{15,16}$ Although the importance of an exhalation valve as it directly relates to respirator fit has not been researched, it has been found to improve heat elimination from inside a respirator ${ }^{26,27}$ and overall comfort. ${ }^{28}$ This, in turn, translates to longer periods of wear and increased effectiveness. The inclusion of an exhalation valve was accordingly considered in the effectivity scoring, alongside size availability and the inclusion of a head strap.

As there is no current literature that suggests the relative impact of these features, a basic point system was implemented to score the effectivity of each respirator. A respirator received one full "head strap" point for the presence of a head strap, and one full "exhalation valve" point for the inclusion of at least one exhalation valve. Of all the respirators considered, the respirator that offers the greatest number of sizes earned one full "size" point for sizing availability. A respirator that is only sold as one-size-fits-all received zero "size" points, as the mask does not offer customizability in fit. The head strap point, exhalation valve point, and size point were aggregated for each respirator and the resulting values were adjusted to a five-point scale. It was determined using the following equations:

$$
\begin{aligned}
s_{i} & =\left(n_{i}-1\right) /(N-1) \\
E_{i} & =\left(h_{i}+v_{i}+s_{i}\right) \times \frac{5}{3}
\end{aligned}
$$

where $s_{i}=$ size point for respirator $i ; n_{i}=$ number of available sizes for respirator $i$; $N=$ the greatest number of sizes of any respirator considered; $E_{i}=$ effectivity score of respirator $i ; h_{i}=$ head strap point for respirator $i ; v_{\mathrm{i}}=$ exhalation valve point for respirator $i$.

\section{COST}

Cost data were collected from a variety of sources, including direct communication with manufacturers, the manufacturers' online stores, online retailers, and local Bangkok retail stores and pharmacies. 
The longevity factor of each respirator affects end-cost. The two time frames (haze period and year-long period) and two order sizes (individual and bulk) translate into four distinct cost scenarios per respirator model. End-cost score was determined within each scenario, and is relative to the highest scoring respirator. The lowest costing respirator received five points, and the end-costs of all other respirators were scaled relative to this respirator with the lowest endcost. As such, the cost of respirators are negatively correlated with their cost scores.

\section{ENVIRONMENTAL IMPACT}

To assess the potential environmental impacts of particulate respirators examined in this integrated assessment, the study used the ReCiPe impact assessment method within the SimaPro 8 Life Cycle Assessment software (PRé Sustainability, Amersfoort, NL). ${ }^{29,30}$ ReCiPe evaluates characterization factors at the midpoint and endpoint level; midpoint indicators focus on single environmental impacts and endpoint indicators show the environmental impact at higher aggregation levels. Although aggregation increases uncertainty and decreases specificity of the results, the simplification of characterization factors allows the results to be better understood by the general public. This study assessed respirators based on the three ReCiPe endpoints: damage to human health, damage to ecosystems, and damage to resource availability. A 2014 field study surveyed Thai people about their perceived importance of these 3 endpoint indicators, and found that the general public opinion mirrors the hierarchical approach. ${ }^{31}$ As such, this Life Cycle Assessment used the hierarchical cultural approach to determine a single index that will quantify the overall environmental impact of a given respirator model. The environmental impact score for each respirator is represented by this single index.

The three phases of product life considered in this study are defined as: material production, respirator use, and respirator disposal. A product distribution phase was originally taken into account, however, global transport of respirators contributed to less than $2 \%$ of overall impacts of the product life cycle. Product distribution is therefore excluded from the study.

As with cost, environmental impact varies between temporal scenarios-haze and year-long protection-as different lengths of protection from ambient air quality require different numbers of respirators, depending on the longevity factor. The longer a particulate respirator's longevity factor, the smaller impact the product may have on the environment.

To collect data for the material production phase, the average mass of each material component per respirator was collected by disassembling three units of each respirator type. The components were massed and averaged; impacts were assessed using the ecoinvent 3 life cycle inventory database. ${ }^{29,32}$ The packaging materials and their masses were assumed to be approximately equivalent across all respirators. Under this assumption, packaging would not alter the relative environmental impact scores of a given respirators in any scenario; as a result, the packaging of the respirators was not considered in the study.
For the purposes of this study, "use" is defined as the wearing and maintenance of a particulate respirator; to maintain reusable respirators, they may be washed. Manufacturers recommend semi-frequent hand-washing with soap and water for hygienic reasons; it does not affect the function of the respirator. The study assumed that reusable respirators are washed once every two weeks. Reusable respirators will therefore be washed one time during an average haze period and 26 times throughout the entire year. It is assumed that 1.5 liters of deionized tap water and one milliliter of general use soap are used to wash each reusable respirator. ${ }^{3}$ Disposable respirators do not require maintenance and thus do not accumulate impacts during the use phase.

Finally, the environmental impacts associated with the disposal phase of each respirator were considered. Bangkok's waste treatment mainly consists of landfill disposal and recycling. It was assumed that approximately 20 $\%$ of annual generated household waste in Thailand is recycled. Respirator model RR4 is entirely recyclable; as such, it was assumed that approximately $20 \%$ of model RR4 is recycled. This study also assumed that $100 \%$ of all non-recyclable respirators are disposed of in landfills; the emissions associated with municipal solid waste collection services for $150 \mathrm{~km}$ transport to a Bangkok landfill are accounted for.

The respective impacts from the three product life cycle phases were compiled into a single score for each respirator. The respirator with the lowest single score, associated with the fewest environmental burdens, received the highest environmental impact score (five points). The scores of the other respirators were scaled relative to this respirator with the lowest environmental impact. As such, the environmental burdens of respirators are negatively correlated with their environmental impact scores.

\section{STEP 2: SCORE AGGREGATION}

Each scenario's three criteria scores were ultimately aggregated into one final score on a five-point scale per respirator. These final integrated scores indicated a top performing respirator within each consumer scenario (A-D), with one of three purchasing models in mind: low-cost, sustainable, or controlled purchasing.

Across all twelve frameworks, respirator effectivity comprised $40 \%$ of the integrated score, given that effective filtration is the primary function of a particulate respirator regardless of consumer preferences. As shown in Figure 4, in the low cost model, cost contributed $40 \%$ of the integrated score. Similarly, in the sustainability model, the environmental impact score contributed $40 \%$. For the control model, cost and environmental impact scores contributed an equal $30 \%$.

\section{RESULTS}

The individual criteria scores are first assessed prior to aggregation: 
Table 3. Overall effectivity scores

\begin{tabular}{|l|c|c|}
\hline & \multicolumn{2}{|c|}{ All Scenarios } \\
\hline Respirator & Effectivity & Score \\
\hline RR1 & 3.00 & 5.00 \\
\hline RR2 & 2.00 & 3.33 \\
\hline RR3 & 1.67 & 2.78 \\
\hline RR4 & 1.33 & 2.22 \\
\hline DR1 & 1.17 & 1.94 \\
\hline DR2 & 2.17 & 3.61 \\
\hline DR3 & 2.00 & 3.33 \\
\hline
\end{tabular}

\section{CRITERIA SCORES}

\section{EFFECTIVITY}

An effectivity score was determined for each particulate respirator and applied to all consumer scenarios A-D, as shown in Table 3 . Out of three total points, the average total effectivity for reusable respirators was 2.0 points; the average total effectivity for disposable respirators is 1.8 . The reusable respirators are therefore 1.1 times more effective on average than the disposable respirators considered in this report.

\section{$\operatorname{cosT}$}

Unlike effectiveness, cost scores varied between scenarios (see Table 4). In both haze period scenarios A and C, the average cost of reusable respirators was significantly higher than that of disposable respirators-between 8 and 9 times more expensive. However, over the course of the entire year (scenarios B and D), the average prices of reusable and disposable respirators intersected. 
Table 4. Overall cost scores

\begin{tabular}{|c|c|c|c|c|c|c|c|c|c|c|c|c|}
\hline & \multicolumn{3}{|c|}{ Scenario A } & \multicolumn{3}{|c|}{ Scenario B } & \multicolumn{3}{|c|}{ Scenario C } & \multicolumn{3}{|c|}{ Scenario D } \\
\hline & $\begin{array}{r}\text { Total cost } \\
(\mathrm{THB}) \\
\end{array}$ & $\begin{array}{r}\text { Total cost } \\
\text { (USD) }\end{array}$ & Score & $\begin{array}{r}\text { Total cost } \\
(\mathrm{THB}) \\
\end{array}$ & $\begin{array}{r}\text { Total cost } \\
\text { (USD) }\end{array}$ & Score & $\begin{array}{r}\text { Total cost } \\
(\mathrm{THB}) \\
\end{array}$ & $\begin{array}{r}\text { Total cost } \\
\text { (USD) } \\
\end{array}$ & Score & $\begin{array}{r}\text { Total cost } \\
(\mathrm{THB}) \\
\end{array}$ & $\begin{array}{r}\text { Total cost } \\
\text { (USD) }\end{array}$ & Score \\
\hline RR1 & 2,131 & 69 & 0.11 & 6,301 & 205 & 1.01 & $2,129,291$ & 69,330 & 0.07 & $6,296,960$ & 205,029 & 0.59 \\
\hline RR2 & 390 & 13 & 0.63 & 1,350 & 44 & 4.72 & 214,500 & 6,984 & 0.74 & 742,500 & 24,176 & 5.00 \\
\hline RR3 & 1,328 & 43 & 0.18 & 3,983 & 130 & 1.60 & $1,327,037$ & 43,208 & 0.12 & $3,981,111$ & 129,625 & 0.93 \\
\hline RR4 & 1,698 & 55 & 0.14 & 5,228 & 170 & 1.22 & 568,891 & 18,523 & 0.28 & $1,607,051$ & 52,326 & 2.31 \\
\hline DR1 & 49 & 2 & $\underline{5.00}$ & 1,274 & 41 & $\underline{5.00}$ & 31,717 & 1,033 & $\underline{5.00}$ & 824,644 & 26,850 & 4.50 \\
\hline DR2 & 106 & 3 & 2.31 & 2,753 & 90 & 2.31 & 69,777 & 2,272 & 2.27 & $1,814,194$ & 59,070 & 2.05 \\
\hline DR3 & 340 & 11 & 0.72 & 8,835 & 288 & 0.72 & 250,677 & 8,162 & 0.63 & $6,517,594$ & 212,213 & 0.57 \\
\hline
\end{tabular}

The conversion rate of 30.7126 THB to 1 USD (July 31, 2019) was used to determine the above values. 
Table 5. Overall environmental impact scores

\begin{tabular}{|l|c|c|c|c|}
\hline & \multicolumn{2}{|c|}{ Haze Period } & \multicolumn{2}{c|}{ Year period } \\
\hline Respirator & Impact & Score & Impact & Score \\
\hline RR1 & 12.49 & 2.59 & 91.22 & 2.16 \\
\hline RR2 & 7.62 & 4.25 & 49.53 & 3.98 \\
\hline RR3 & 8.60 & 3.77 & 49.78 & 3.96 \\
\hline RR4 & 7.10 & 4.56 & 39.44 & $\underline{5.00}$ \\
\hline DR1 & 6.47 & $\underline{5.00}$ & 168.29 & 1.17 \\
\hline DR2 & 10.03 & 3.23 & 260.84 & 0.76 \\
\hline DR3 & 9.56 & 3.39 & 248.45 & 0.79 \\
\hline
\end{tabular}

\section{ENVIRONMENTAL IMPACT}

The most environmentally-sustainable respirator model differed between short-term and long-term use periods. As shown in Table 5, model DR1 received the highest environmental impact score within the short-term use scenario. Model RR4 received the highest score within for long-term use. The environmental impact scores of reusable respirators and disposable respirators for short term use had little variance. Although, in the year-long scenarios, the average reusable and disposable impact values quickly diverged. Disposable respirators were associated with much higher environmental impacts over an entire year than reusable respirators-after 52 weeks, the average disposable respirator had over 3.8 times as much of an impact on the environment as does the average reusable respirator.

\section{FINAL INTEGRATED SCORES}

The final integrated scores aimed to provide results to a wide range of consumers with distinct purchasing plans. Table 6 (scenarios A-D) lists the integrated scores of all seven respirator models within each of the twelve consumer frameworks. In summary, for both short-term consumer scenarios $(A, C)$, the data suggest respirator model DR1 as the optimal respirator model. Model DR1 is a disposable respirator with a head strap and one or two available sizes; this model costs THBB30 (USD\$0.97) per respirator. For both long-term scenarios (B, D), the data suggest respirator model RR2 as the optimal respirator model. Model RR2 is a reusable respirator with replaceable filters, a head strap, an exhalation valve, and four available sizes; this model costs THBB400 (USD\$12.96) per respirator.

\section{DISCUSSION}

As derived from Table 3, model RR1 was the most effective respirator evaluated because it fulfilled all fit features outlined by the effectivity scoring guidelines. Reusable models RR3 and RR4 scored relatively low in comparison to the other reusable respirators, as RR3 does not include a head strap, and RR4 does not include valves. The average reusable respirator is 1.1 times more effective than the av- erage disposable respirator when assessed by the facial fit standards established in this study. The slight variation in relative effectivity of particulate respirator types was attributable to the fact that each of the reusable respirators evaluated offers more than one size to fit a range of consumer types, while disposable respirators often do not. The additional sizes contributed to a higher effectivity score, and what a consumer may therefore understand as a better fit.

Directly comparing the disposable respirator models DR1 and DR2, the effectiveness of DR2 nearly doubled that of DR1. These models are nearly identical, aside from the inclusion of an exhalation valve, and hence the importance of the valve feature is reinforced. It is also significant to note that respirator models DR2 and DR3 had the same or higher effectivity scores that RR2, RR3, and RR4. However, DR2 and DR3 had a much lower upfront cost in comparison to the reusable respirators. A consumer can conclude that a high cost does not necessarily represent a high efficiency. Instead, the consumer should seek the best-fitting respirator, regardless of cost.

As a final note on effectivity: if the average surgical mask were to be scored by these guidelines, the mask would receive zero points for effectivity. Aside from the fact that surgical masks are not approved to filter 95\% of PM2.5, they typically lack multiple sizes, head straps, and exhalation valves. Surgical masks should not be considered for the effective filtration of fine particulate matter.

As illustrated in Table 4, the lowest cost respirator option for consumer scenarios A-C is DR1, the simplest of the seven respirator models. Model DR1 is a disposable hard-shell cup respirator with no exhalation valve. The average upfront cost of a DR1 respirator is THBB25 (USD\$0.81), and significant discounts are often applied to bulk orders from the manufacturer. For scenario D, the least expensive model is RR2, which is the low-cost reusable respirator with an upfront cost of THBB390 (USD\$12.70). Although disposable respirators cost significantly less for short-term purchasing periods, the average prices of reusable and disposable respirators intersect over time. The cost difference between the RR2 and DR1 for the individual consumer after one haze period was roughly THBB340 (USD\$11.10), RR2 being the more expensive model. However, the overall cost difference between the RR2 and DR1 for the individual consumer after 
Table 6. Final integrated scores: scenarios A, B, C, and D

\begin{tabular}{|c|c|c|c|}
\hline \multirow[b]{2}{*}{ Respirator } & \multicolumn{3}{|c|}{ Purchasing model } \\
\hline & Control & Low-cost & Sustainable \\
\hline \multicolumn{4}{|l|}{ Scenario A } \\
\hline RR1 & 2.56 & 3.06 & 2.81 \\
\hline RR2 & 2.43 & 3.16 & 2.80 \\
\hline RR3 & 1.94 & 2.66 & 2.30 \\
\hline RR4 & 1.86 & 2.74 & 2.30 \\
\hline DR1 & $\underline{3.78}$ & $\underline{3.78}$ & $\underline{3.78}$ \\
\hline DR2 & 3.01 & 3.20 & 3.11 \\
\hline DR3 & 2.30 & 2.83 & 2.57 \\
\hline \multicolumn{4}{|l|}{ Scenario B } \\
\hline RR1 & 2.84 & 3.07 & 2.95 \\
\hline RR2 & $\underline{4.02}$ & $\underline{3.87}$ & $\underline{3.94}$ \\
\hline RR3 & 2.54 & 3.02 & 2.78 \\
\hline RR4 & 2.28 & 3.08 & 2.68 \\
\hline DR1 & 3.01 & 2.24 & 2.63 \\
\hline DR2 & 2.52 & 2.21 & 2.37 \\
\hline DR3 & 1.78 & 1.79 & 1.79 \\
\hline \multicolumn{4}{|l|}{ Scenario C } \\
\hline RR1 & 2.55 & 3.05 & 2.80 \\
\hline RR2 & 2.48 & 3.18 & 2.83 \\
\hline RR3 & 1.91 & 2.64 & 2.28 \\
\hline RR4 & 1.91 & 2.77 & 2.34 \\
\hline DR1 & $\underline{3.78}$ & $\underline{3.78}$ & $\underline{3.78}$ \\
\hline DR2 & 3.00 & 3.19 & 3.09 \\
\hline DR3 & 2.26 & 2.81 & 2.54 \\
\hline \multicolumn{4}{|l|}{ Scenario D } \\
\hline RR1 & 2.67 & 2.98 & 2.82 \\
\hline RR2 & $\underline{4.13}$ & $\underline{3.93}$ & $\underline{4.03}$ \\
\hline RR3 & 2.28 & 2.88 & 2.58 \\
\hline RR4 & 2.81 & 3.35 & 3.08 \\
\hline DR1 & 2.81 & 2.14 & 2.48 \\
\hline DR2 & 2.42 & 2.16 & 2.29 \\
\hline DR3 & 1.72 & 1.76 & 1.74 \\
\hline
\end{tabular}

Scenario A describes an "individual order" of 1 respirator over the course of one haze period.

Scenario B describes an "individual order" of 1 respirator over the course of an entire year.

Scenario C describes a "bulk order" of 1,000 respirators over the course of one haze period.

Scenario D describes a "bulk order" of 1,000 respirators over the course of an entire year.

one year was roughly THBB80 (USD\$2.59), RR2 still being slightly more expensive, but by a much smaller margin.

A consumer can conclude that if he or she would like to purchase a respirator (or distribute respirators), the disposable models are the most cost-effective option. Only after 1.5 years does the cost of disposable and reusable models intersect; this would be because the consistent replacement of disposable respirators eventually becomes more expensive than reusable respirators. The shorter longevity factors of disposable respirators mean that more products must be purchased per time period in comparison to reusable respirators.

The environmental impact scores of particulate respirators varied to a greater extent over long durations of time (Table 5). Because of the short longevity factor of disposable respirators, fifty-two units must be purchased over the entire year to protect an individual from fine particulates. The highest number of reusable respirators required for an entire year is three units. The high number of disposable respirators cause far more emissions to the environment due 
to the additional materials, energy, transportation, and other factors needed to support long-term use. Reusable models may therefore be considered the more environmentally sustainable respirator over time.

Model RR4, the most sustainable respirator for longterm use, is a reusable respirator made from the fewest number of materials of the reusable respirators, most of which are recyclable. Only the respirator shell needs to be replaced after two years of use if properly maintained. Although the respirator requires replacement filters and routine washing throughout the year, the impacts associated with this respirator are still comparatively very small. Similar conclusions can be made regarding the other reusable respirators. Although none of the other reusable respirator models may be recycled after use, their high longevity factors result in fewer impacts on the environment. As stated in the results, the average disposable respirator has an environmental impact that is almost four times greater than the average reusable respirator. For the long-term consumer with sustainable purchasing preferences, a reusable respirator will always be the optimal choice.

For both reusable and disposable respirator models, the majority of all environmental impacts associated with short term use are attributable to the material production phase of the product life cycle-roughly $85 \%$. The use phase contributes to over $12 \%$ of the impacts of reusable models after one wash. After the entire year (twenty-six total washes), the use phase contributes to around $80 \%$ of total product impact. The impacts associated with the long-term use of reusable respirators is attributable to the washing maintenance-while disposable respirators do not generate impacts in this specific life cycle phase. The waste phase for both respirator types contributes to roughly $9 \%$ of the single scores. One way to decrease the impacts associated with the waste phase of a particulate respirator is to select a product that has at least some parts that are recyclable. As an example, over $13 \%$ of the environmental impacts associated with the model RR4 are reduced due to the model's recyclability, making it one of the most environmentally sustainable model choices in both temporal scenarios.

There were apparent trends in the data regarding the final integrated scores of the seven particulate respirator models. For short term-purchasing, disposable respirators had the highest scores. The scores for DR1 topped all other scores within all three purchasing models for consumer scenarios $\mathrm{A}$ and $\mathrm{C}$. The extremely low cost and small environmental impact of the DR1 model were the factors that most significantly supported its high scores in the haze period scenarios. However, it is significant to note that respirator model DR1 scored lowest in effectivity. Although this model is an optimal respirator choice by the standards set in this study, it is essential to acknowledge its potential flaws; DR1 often does not come in a range of sizes, nor does it include an exhalation valve. Therefore, although they may suit the average user, DR1 models that are one-size-only are unlikely to perform well on individuals with smaller faces, such as children. This assessment recommends DR1 only after the consideration of the target wearer. For example, if a federal agency wanted to provide respirators for primary schools, it would be best to select a respirator with better fit characteristics. Having even two respirator sizes (small and large) compared to one size (large) will increase the models effectivity by $33 \%$.

For long term-use (scenarios B and D), respirator model RR2 outperformed all other models. RR2 is a low-cost reusable respirator with an exhalation valve and replaceable filters. Although this is not the most effective or sustainable model, it scored within the mid to high ranges of these individual criteria. The high integrated score of the RR2 model is most attributable to its low cost in relation to other reusable respirators. The average upfront cost of a reusable respirator is about THBB1,400 (USD\$45.38)-the upfront cost of RR2 is only THBB390 (USD\$12.70). Additionally, the RR2 model can last an entire year when properly maintained because of its replaceable filters and ability to be washed. It did not score a full 3 points by the effectivity fit-scoring guidelines because the model does not come with a head strap, however one can be purchased separately. The respirator does come in four different sizes, allowing effective use to a range of user-types. The results suggest that consumers, individual or bulk, should consider lowcost reusable respirators with replaceable filters and a range of available sizes if they seek year-long protection from fine particulate matter.

\section{CONCLUSIONS}

The goal of this study was to suggest the optimal respirator for consumers with diverse lifestyles, preferences, and purposes in the metropolises of Thailand. This was done by way of a multi-part assessment that integrated data on each respirator's effectivity, cost, and environmental impacts. Seven respirator models were evaluated: four reusable, and three disposable. These data were scored and aggregated based on consumer frameworks designed for, and outlined in, this study. Within each scenario, respirators were scored using three purchasing models: low-cost, sustainable, and controlled. Despite variations in relative scores, the highest scoring respirator remained consistent across consumer frameworks, regardless of the weights used for the three scoring criteria. For scenarios that target individual and bulk consumers buying for respiratory protection during a short-term haze period, the assessment recommends the lowest cost disposable respirator model with a head strap and multiple sizes available. For scenarios that target individual and bulk consumers with long-term purchasing plans, the assessment recommends the lowest cost reusable respirator with replaceable filters, exhalation valves, and multiple sizes offered. Future research could further investigate consumer habits to detail the public's opinion of respirator efficiency, cost and environmental sustainability. This information would assist in making a more accurate framework to represent the average Thai consumer. Finally, a cost benefit analysis between respirator use and hospitalizations would be very useful for policy makers, to assist in making decisions that prioritize both public health and fiscal responsibility.

Acknowledgements: The authors would like to thank the 
Life Cycle Sustainability Assessment Laboratory at the JGSEE (Joint Graduate School of Energy and Environment) at KMUTT (King Mongkut's University of Technology Thonburi, Thailand) for sharing their expertise with, and access to, the life cycle assessment software, the UNC Institute for the Environment for sponsoring the UNC Thailand field site hosted at KMUTT, and the JGSEE at KMUTT for providing a laboratory in which to perform scientifically sound research. The authors would also like to thank Dr. Thao Pham, Prof. Richard Kamens, Dr. Savitri Garivait, and Sitthipong Pengjan for constructive discussions throughout this research.

Funding: This research received financial support from the JGSEE at KMUTT for the purpose of purchasing particulate respirators.

Authorship contributions: KLM, KEW, and IRH conceived of the presented idea. KLM, KEW, and IRH performed the com- putations and LCA of each particulate respirator, under the guidance and intellectual support of SHG. KLM, KEW, and IRH wrote the manuscript. SHG contributed significant suggestions to the methodology of the research, and revisions to the manuscript.

Competing interests: The authors completed the Unified Competing Interest form at www.icmje.org/coi_disclosure.pdf (available upon request from the corresponding author), and declare no conflicts of interest.

\section{Correspondence to:}

Shabbir H. Gheewala, D.Eng., Professor

The Joint Graduate School of Energy and Environment King Mongkut's University of Technology Thonburi 126 Pracha Uthit Road, Bangmod, Tungkru, Bangkok 10140, Thailand

shabbir_g@igsee.kmutt.ac.th; shabbirg@hotmail.com 


\section{REFERENCES}

1. World Health Organization. Air Quality Guidelines: Global Update 2005 : Particulate Matter, Ozone, Nitrogen Dioxide, and Sulfur Dioxide. Geneva, Switzerland: World Health Organization; 2006. http s://books.google.com/books/about/Air_Quality_Guide lines.html?hl=\&id=7VbxUdlJE8wC.

2. Narita D, Oanh NTK, Sato K, et al. Pollution Characteristics and Policy Actions on Fine Particulate Matter in a Growing Asian Economy: The Case of Bangkok Metropolitan Region. Atmosphere. 2019;10(5):227. doi:10.3390/atmos10050227

3. Hills S, Birks R, McKenzie B. The Millennium Dome "Watercycle" experiment: To evaluate water efficiency and customer perception at a recycling scheme for 6 million visitors. Water Sci Technol. 2002;46(6-7):233-240. doi:10.2166/wst.2002.0684

4. Thailand Pollution Control Department. Booklet on Thailand State of Pollution 2018. Ministry of Natural Resources and Environment; 2019.

5. Attavanich W. Air Pollution in Bangkok: Status, Causes \& Economic Effects. Presented at the: PIER Economic Seminar; February 11, 2019; Bank of Thailand, Bangkok. https://www.pier.or.th/wp-conten t/uploads/2019/02/seminar2019 WitsanuAttavanich.p df.

6. Vichit-Vadakan N, Vajanapoom N. Health impact from air pollution in Thailand: Current and future challenges. Environ Health Perspect. 2011;119(5):A197-8. doi:10.1289/ehp.1103728

7. Xing Y-F, Xu Y-H, Shi M-H, et al. The impact of PM2.5 on the human respiratory system. J Thorac Dis. 2016;8(1):E69-74. http://dx.doi.org/10.3978/j.issn.207 2-1439.2016.01.19.

8. Xu X, Ha SU, Basnet R. A Review of Epidemiological Research on Adverse Neurological Effects of Exposure to Ambient Air Pollution. Front Public Health. 2016;4:157. doi:10.3389/fpubh.2016.00157

9. WHO. How air pollution is destroying our health. 2018. https://www.who.int/air-pollution/news-and-ev ents/how-air-pollution-is-destroying-our-health.

10. "Unhealthy for Sensitive Groups" Air-Quality Index. Clean Air in the River Valley: Environmental Education, Technology, Partnerships and Planning. The University of Iowa.
11. Pope CA. Epidemiology of Fine Particulate Air Pollution and Human Health: Biologic Mechanisms and Who's at Risk? Environ Health Perspect. 2000;108(s4):713-723. doi:10.1289/ehp.00108s4713

12. Langrish JP, Mills NL, Chan JK, et al. Beneficial cardiovascular effects of reducing exposure to particulate air pollution with a simple facemask. Part Fibre Toxicol. 2009;6(1):8. doi:10.1186/1743-8977-6-8

13. Occupational Safety and Health Administration. Respiratory Protection. United States Department of Labor; 2019.

14. Zhuang Z, Bergman M, Lei Z, Niezgoda G, Shaffer R. Recommended test methods and pass/fail criteria for a respirator fit capability test of half-mask airpurifying respirators. J Occup Environ Hyg. 2017;14(6):473-481. doi:10.1080/15459624.2017.1296 $\underline{233}$

15. Zhuang Z, Ann RB, Viscusi D. 224. The Effect of Subject Characteristics and Respirator Features on Respirator Fit. In: AIHce 2003. AIHA; 2003:224. doi:1 $\underline{0.3320 / 1.2757902}$

16. Cherrie JW, Apsley A, Cowie H, et al. Effectiveness of face masks used to protect Beijing residents against particulate air pollution. Occup Environ Med. 2018;75(6):446-452. doi:10.1136/oemed-2017-104765

17. Viscusi DJ, Bergman MS, Zhuang Z, Shaffer RE. Evaluation of the benefit of the user seal check on N95 filtering facepiece respirator fit. J Occup Environ Hyg. 2012;9(6):408-416. doi:10.1080/15459624.2012.6 $\underline{83757}$

18. Google Trends. http://www.google.com/trends/. Published 2014. Accessed July 16, 2019.

19. Noomnual S, Shendell DG. Young Adult Street Vendors and Adverse Respiratory Health Outcomes in Bangkok, Thailand. Saf Health Work.

2017;8(4):407-409. doi:10.1016/j.shaw.2017.02.002

20. Lee S-A, Grinshpun SA, Reponen T. Respiratory performance offered by $\mathrm{N} 95$ respirators and surgical masks: Human subject evaluation with $\mathrm{NaCl}$ aerosol representing bacterial and viral particle size range. Ann Occup Hyg. 2008;52(3):177-185. doi:10.1093/ann $\underline{\text { hyg } / \text { men005 }}$ 
21. Grinshpun SA, Haruta H, Eninger RM, Reponen T, McKay RT, Lee S-A. Performance of an N95 filtering facepiece particulate respirator and a surgical mask during human breathing: Two pathways for particle penetration. J Occup Environ Hyg. 2009;6(10):593-603. doi:10.1080/15459620903120086

22. Lee S-A, Hwang D-C, Li H-Y, Tsai C-F, Chen C-W, Chen J-K. Particle Size-Selective Assessment of Protection of European Standard FFP Respirators and Surgical Masks against Particles-Tested with Human Subjects. J Healthc Eng. 2016;2016:1-12. doi:10.1155/ $\underline{2016 / 8572493}$

23. Bangkok Air Pollution: Real-Time Air Quality Index (AQI). Bangkok: World Air Quality Index Project; 2019.

24. Air Quality Index Scale and Color Legend. World Air Quality Index Project; 2019.

25. Smog forces all Bangkok schools to close. Bangkok Post. https://www.bangkokpost.com/thailand/genera 1/1620482/smog-forces-all-bangkok-schools-to-close. Published January 30, 2019.

26. Mu Q, Zhang J. Air Pollution and Defensive Expenditures: Evidence from Particulate-Filtering Facemasks. SSRN Journal. 2014. doi:10.2139/ssrn.251 $\underline{8032}$
27. Roberge RJ. Are exhalation valves on N95 filtering facepiece respirators beneficial at low-moderate work rates: An overview. J Occup Environ Hyg. 2012;9(11):617-623. doi:10.1080/15459624.2012.7150 $\underline{66}$

28. Seng M, Wee L, Zhao X, Cook A, Chia S, Lee V. Comfort and exertion while using filtering facepiece respirators with exhalation valve and an active venting system among male military personnel. Singapore Med J. 2018;59(6):327-334. doi:10.11622/s medj.2017054

29. Huijbregts MAJ, Steinmann ZJN, Elshout PMF, et al. ReCiPe2016: A harmonised life cycle impact assessment method at midpoint and endpoint level. Int J Life Cycle Assess. 2017;22(2):138-147. doi:10.100 7/s11367-016-1246-y

30. Wernet G, Bauer C, Steubing B, et al. SimaPro Ecoinvent Database. Int J Life Cycle Assess. 10:3-9.

31. Kuntachaianun N, Tran T, Hansupalak N. Weighting Factors for LCA Impact Indicators by a Panel Approach for Thailand. International Proceedings of Chemical, Biological and Environmental Engineering. 2015;82(18):94-98. doi:1 $\underline{0.7763 / \text { ipcbee }}$

32. Wernet G, Bauer C, Steubing B, Reinhard J, Moreno-Ruiz E, Weidema B. The ecoinvent database version 3 (part I): Overview and methodology. Int J Life Cycle Assess. 2016;21(9):1218-1230. doi:10.1007/ $\underline{\text { s11367-016-1087-8 }}$ 